\title{
SOLUTIONS OF VIBRATION PROBLEMS FOR THIN INFINITE PLATES SUBJECTED TO HARMONIC LOADS
}

\author{
Michael Klanner, Katrin Ellermann \\ Graz University of Technology, Institute of Mechanics, Graz, Austria \\ e-mail:michael.klanner@tugraz.at
}

\begin{abstract}
New closed form solutions for harmonic vibrations of infinite Kirchhoff plates subjected to a constant harmonic ring load, a constant harmonic circular load and an alternating harmonic circular load are derived. Two different approaches are used to define the closed form solutions. The first approach uses the integration of the harmonic point force and the addition theorem for Bessel functions, while the second approach applies the Hankel transform to solve the inhomogeneous partial differential equation of the Kirchhoff plate theory. The new closed form particular solutions can especially be used in Trefftz like methods and extend their field of application.
\end{abstract}

Keyword: Kirchhoff plate theory, infinite plate, ring load, circular load, Hankel transform

\section{Introduction}

The simulation of vibrations and emitted sound of plates is an important step in the development of new products, since the noise and vibration characteristics have to be considered in an early design phase. The most common method to simulate vibrations of plates is the Finite Element Method (FEM) (Bathe, 2006), which is especially well applicable for low frequency vibrations. In the recent years, several methods which use the concept presented by Trefftz in 1926 (Trefftz, 1926), have been developed to calculate vibrations of plates more efficiently for higher frequencies. Among these methods one can mention the Wave Based Method (Vanmaele et al., 2007; Klanner and Ellermann, 2015) and the Variational Theory of Complex Rays (VTCR) (Rouch and Ladevèze, 2003; Riou et al., 2013). In general, the so-called indirect Trefftz methods require a particular solution of the inhomogeneous partial differential equation to be applied efficiently. This is the motivation to develop new closed form solutions for the vibrations of Kirchhoff plates in this paper.

To the authors knowledge, closed form particular solutions for infinite Kirchhoff plates only exist in literature for undamped plates excited by a concentrated point force, which can be found in e.g. (Junger and Feit, 1986).

The Kirchhoff plate theory was introduced by Kirchhoff in 1850 (Kirchhoff, 1850) and neglects rotatory inertia and shear deformation. Therefore, it is only applicable if the ratio of the plate thickness to the lesser of the other two dimensions is smaller than $1: 20$ (Chandrashekhara, 2001) and the ratio of the plate thickness to the bending wave length is smaller than $1: 6$ (Cremer et al., 2005). In many practical problems, these limits are fulfilled and the Kirchhoff plate model can be used very sufficiently compared to a full 3D model.

The paper is structured in Sections as follows: The particular solution of an infinite Kirchhoff plate excited by a concentrated point force in the case of a damped plate is derived in Section 2 . In Section 3, other axisymmetric load cases, the constant ring load and the constant circular load, are considered and two different approaches are shown to determine the closed form solutions. In Section 4, a non-axisymmetric load is considered, which represents an alternating circular 
load. The vibrations in the case of an undamped plate are derived in Section 5. The responses to the mentioned excitations are plotted in Section 6 for an aluminum plate. Finally, a conclusion is given and further research topics are discussed.

\section{Point force excitation}

In this Section, the governing equation of the Kirchhoff plate theory is stated and the governing equation in the special case of an axisymmetric vibration is shown. The Hankel transform of the order zero is briefly presented and the closed form solution for the Kirchhoff plate under harmonic point force excitation is derived.

\subsection{Governing equation for time harmonic problems}

The governing equation of the Kirchhhoff plate theory for time harmonic vibrations in Cartesian coordinates is given by (Rao, 2007)

$$
\nabla^{4} w(x, y)-k_{b}^{4} w(x, y)=\frac{q(x, y)}{D}
$$

where $\nabla^{4}=\frac{\partial^{4}}{\partial x^{4}}+2 \frac{\partial^{4}}{\partial x^{2} \partial y^{2}}+\frac{\partial^{4}}{\partial y^{4}}$, the bending wavenumber $k_{b}^{4}=\rho h \omega^{2} / D$ and the plate bending stiffness $D=E h^{3} /\left[12\left(1-\nu^{2}\right)\right], E$ is Young's modulus, $h$ - plate thickness, $\rho$ - plate density, $\nu$ Poisson's ratio, $\omega$ - angular frequency and $q(x, y)$ - external force distribution. For axisymmetric problems, e.g. the point force excitation, the governing equation in polar coordinates

$$
\left(\frac{d^{2}}{d r^{2}}+\frac{1}{r} \frac{d}{d r}\right)^{2} w(r)-k_{b}^{4} w(r)=\frac{q(r)}{D}
$$

depends only on the radius $r$. Using a complex elastic modulus introduces damping of the plate, and the plate parameters become complex

$$
E \rightarrow \widetilde{E}=E(1+\mathrm{i} \eta) \quad D \rightarrow \widetilde{D}=D(1+\mathrm{i} \eta) \quad k_{b}^{4} \rightarrow \widetilde{k}_{b}^{4}=\frac{k_{b}^{4}}{1+\mathrm{i} \eta}=k_{b d}^{4} \mathrm{e}^{-\mathrm{i} \Phi}
$$

with

$$
k_{b d}^{4}=\frac{k_{b}^{4}}{\sqrt{1+\eta^{2}}} \quad k_{b d}^{4} \in \mathbb{R}^{+} \quad \Phi=\arctan \eta \quad 0<\Phi<\frac{\pi}{2}
$$

and $\eta>0$ the material loss factor.

\subsection{Hankel transform of the order zero}

The Hankel transform of the order zero is defined by (Debnath and Bhatta, 2014)

$$
\widetilde{f}\left(k_{r}\right)=\int_{0}^{\infty} f(r) r J_{0}\left(k_{r} r\right) d r \quad \text { and } \quad f(r)=\int_{0}^{\infty} \widetilde{f}\left(k_{r}\right) k_{r} J_{0}\left(k_{r} r\right) d k_{r}
$$

with the operational property for the axisymmetric Laplace operator (Debnath and Bhatta, 2014)

$$
\left(\frac{d^{2}}{d r^{2}}+\frac{1}{r} \frac{d}{d r}\right) f(r) \mapsto-k_{r}^{2} \tilde{f}\left(k_{r}\right)
$$

In the case of axisymmetric vibrations, the governing equation of the Kirchhoff plate (Eq. (2.2)) transforms to

$$
\widetilde{w}\left(k_{r}\right)=\frac{1}{\widetilde{D}} \frac{\widetilde{q}\left(k_{r}\right)}{k_{r}^{4}-\widetilde{k}_{b}^{4}}
$$




\subsection{Point load at the origin of the coordinate system}

A point load at the origin of the coordinate system $(x=0, y=0$ or $r=0)$ is given by

$$
q_{\text {point }}(x, y)=q_{0} \delta(x) \delta(y) \Rightarrow q_{\text {point }}(r)=\frac{q_{0} \delta(r)}{2 \pi r} \mapsto \widetilde{q}_{\text {point }}\left(k_{r}\right)=\frac{q_{0}}{2 \pi}
$$

with $q_{0}$ being the amplitude of the harmonic point load. The inverse Hankel transform leads to the solution for the out-of-plane displacement in an integral form

$$
w_{\text {point }}(r)=\frac{q_{0}}{2 \pi \widetilde{D}} \int_{0}^{\infty} \frac{k_{r}}{k_{r}^{4}-\widetilde{k}_{b}^{4}} J_{0}\left(k_{r} r\right) d k_{r}
$$

To obtain a closed form solution, the integral representation of the Bessel function of the first kind (Watson, 1944)

$$
J_{0}\left(k_{r} r\right)=\frac{2}{\pi} \int_{0}^{\infty} \sin \left(k_{r} r \cosh u\right) d u
$$

is used in Eq. (2.9). Interchanging the order of integraton leads to

$$
w_{\text {point }}(r)=\frac{q_{0}}{\pi^{2} \widetilde{D}} \int_{0}^{\infty}\left(\int_{0}^{\infty} \frac{k_{r}}{k_{r}^{4}-\widetilde{k}_{b}^{4}} \sin \left(k_{r} r \cosh u\right) d k_{r}\right) d u
$$

The integral is symmetric with respect to $k_{r}$ and, therefore, can be written as

$$
w_{\text {point }}(r)=\frac{\mathrm{i} q_{0}}{2 \pi^{2} \widetilde{D}} \int_{0}^{\infty}\left(\int_{-\infty}^{\infty} \frac{k_{r}}{k_{r}^{4}-\widetilde{k}_{b}^{4}} \mathrm{e}^{-\mathrm{i} k_{r} r \cosh u} d k_{r}\right) d u
$$

since the integral of an odd function over a symmetric interval vanishes. The integral with respect to $k_{r}$ can be performed using the residue theorem and Jordan's lemma (Mitrinović and Kečkić, 1984). The integrand in Eq. (2.12) has first order poles, if $k_{r}^{4}-\widetilde{k}_{b}^{4}=0$, which leads to the four poles

$$
\begin{array}{lll}
k_{r 1}=k_{b d} \mathrm{e}^{-\mathrm{i} \frac{\Phi}{4}} & \text { with } & \operatorname{Im}\left(k_{r 1}\right)<0 \\
k_{r 2}=-k_{b d} \mathrm{e}^{-\mathrm{i} \frac{\Phi}{4}} & \text { with } & \operatorname{Im}\left(k_{r 2}\right)>0 \\
k_{r 3}=\mathrm{i} k_{b d} \mathrm{e}^{-\mathrm{i} \frac{\Phi}{4}} & \text { with } & \operatorname{Im}\left(k_{r 3}\right)>0 \\
k_{r 4}=-\mathrm{i} k_{b d} \mathrm{e}^{-\mathrm{i} \frac{\Phi}{4}} & \text { with } & \operatorname{Im}\left(k_{r 4}\right)<0
\end{array}
$$

with

$$
k_{b d}=\sqrt[4]{\frac{k_{b}^{4}}{\sqrt{1+\eta^{2}}}}
$$

According to the residue theorem and Jordan's lemma (Mitrinović and Kečkić, 1984)

$$
\int_{-\infty}^{\infty} f(x) \mathrm{e}^{\mathrm{i} a x} d x= \begin{cases}2 \pi \mathrm{i} \sum_{k=1}^{s^{+}} \operatorname{Res}\left[f(z) \mathrm{e}^{\mathrm{i} a z}\right]+\pi \mathrm{i} \sum_{k=1}^{m} \operatorname{Res}_{z=p_{k}}\left[f(z) \mathrm{e}^{\mathrm{i} a z}\right] & \text { for } \quad a>0 \\ -2 \pi \mathrm{i} \sum_{k=1}^{s^{-}} \operatorname{Res}_{z=z_{k}^{-}}\left[f(z) \mathrm{e}^{\mathrm{i} a z}\right]-\pi \mathrm{i} \sum_{k=1}^{m} \operatorname{Res}_{z=p_{k}}\left[f(z) \mathrm{e}^{\mathrm{i} a z}\right] & \text { for } \quad a<0\end{cases}
$$


with $z_{k}^{+}$poles in the upper half plane, $z_{k}^{-}$poles in the lower half plane, $p_{k}$ poles on the real axis and Res[.] for the residue at the pole. The residue for simple poles is given by (Mitrinović and Kečkić, 1984)

$$
\operatorname{Res}_{z=z_{k}}\left[f(z) \mathrm{e}^{\mathrm{i} a z}\right]=\lim _{z \rightarrow z_{k}}\left(z-z_{k}\right) f(z) \mathrm{e}^{\mathrm{i} a z}
$$

In the integral of Eq. (2.12) the function $f(z)=z /\left(z^{4}-\widetilde{k}_{b}^{4}\right)$ and the parameter $a=-r \cosh u<0$ and, therefore, according to Eq. (2.15) 2 , only the poles $k_{r 1}$ and $k_{r 4}$ with the negative imaginary part contribute to the integral. The residues for these two poles are

$$
\operatorname{Res}_{z=k_{r 1}}\left[f(z) \mathrm{e}^{\mathrm{i} a z}\right]=\frac{\mathrm{e}^{-\mathrm{i} r \cosh (u) \widetilde{k}_{b}}}{4 \widetilde{k}_{b}^{2}} \quad \operatorname{Res}_{z=k_{r 4}}\left[f(z) \mathrm{e}^{\mathrm{i} a z}\right]=-\frac{\mathrm{e}^{-r \cosh (u) \widetilde{k}_{b}}}{4 \widetilde{k}_{b}^{2}}
$$

with

$$
\widetilde{k}_{b}^{2}=k_{b d}^{2} \mathrm{e}^{-\mathrm{i} \frac{\Phi}{2}} \quad \widetilde{k}_{b}=k_{b d} \mathrm{e}^{-\mathrm{i} \frac{\Phi}{4}}
$$

Using Heine's formulas for the integral representation of the Hankel functions (Magnus et al., 1966)

$$
\begin{array}{ll}
H_{0}^{(1)}(z)=-\frac{2 \mathrm{i}}{\pi} \int_{0}^{\infty} \mathrm{e}^{\mathrm{i} z \cosh u} d u & 0<\arg (z)<\pi \\
H_{0}^{(2)}(z)=\frac{2 \mathrm{i}}{\pi} \int_{0}^{\infty} \mathrm{e}^{-\mathrm{i} z \cosh u} d u & -\pi<\arg (z)<0
\end{array}
$$

leads to the final result

$$
w_{\text {point }}(r)=\frac{\mathrm{i} q_{0}}{8 \widetilde{D} \widetilde{k}_{b}^{2}}\left(H_{0}^{(1)}\left(-r \widetilde{k}_{b}\right)+H_{0}^{(2)}\left(-\mathrm{i} r \widetilde{k}_{b}\right)\right)
$$

The result in Eq. (2.20) can be given in different forms using the relations (Abramowitz and Stegun, 1972)

$$
\begin{array}{ll}
K_{0}(z)=\frac{1}{2} \pi \mathrm{i} H_{0}^{(1)}(\mathrm{i} z) & -\pi<\arg (z) \leqslant \frac{1}{2} \pi \\
K_{0}(z)=-\frac{1}{2} \pi \mathrm{i} H_{0}^{(2)}(-\mathrm{i} z) & -\frac{1}{2} \pi<\arg (z) \leqslant \pi \\
H_{0}^{(1)}(\mathrm{i} z)=-H_{0}^{(2)}(-\mathrm{i} z) & -\frac{1}{2} \pi<\arg (z) \leqslant \frac{1}{2} \pi
\end{array}
$$

which leads to

$$
w_{\text {point }}(r)=\frac{q_{0}}{4 \pi \widetilde{D} \widetilde{k}_{b}^{2}}\left(K_{0}\left(\mathrm{i} r \widetilde{k}_{b}\right)-K_{0}\left(r \widetilde{k}_{b}\right)\right)=\frac{\mathrm{i} q_{0}}{8 \widetilde{D} \widetilde{k}_{b}^{2}}\left(H_{0}^{(2)}\left(-\mathrm{i} r \widetilde{k}_{b}\right)-H_{0}^{(2)}\left(r \widetilde{k}_{b}\right)\right)
$$

The displacement at $r=0$ can be computed with the limiting case $r \rightarrow 0$

$$
w_{\text {point }}(0)=\lim _{r \rightarrow 0} w_{\text {point }}(r)=-\frac{\mathrm{i} q_{0}}{8 \widetilde{D} \widetilde{k}_{b}^{2}}
$$




\subsection{Point load at an arbitrary point of the coordinate system}

The out-of-plane displacement $w_{\text {point }}$ depends only on the distance between the response point $(x, y)$ and the point of excitation $(\bar{x}, \bar{y})$, which is given by $r=\sqrt{(x-\bar{x})^{2}+(y-\bar{y})^{2}}$. The out-of-plane displacement of the plate subject to a point force at the drive point $(\bar{x}, \bar{y})$ is, therefore, given by

$$
w_{\text {point }}(x, y)=\frac{\mathrm{i} q_{0}}{8 \widetilde{D} \widetilde{k}_{b}^{2}}\left(H_{0}^{(1)}\left(-\sqrt{(x-\bar{x})^{2}+(y-\bar{y})^{2}} \widetilde{k}_{b}\right)+H_{0}^{(2)}\left(-\mathrm{i} \sqrt{(x-\bar{x})^{2}+(y-\bar{y})^{2}} \widetilde{k}_{b}\right)\right)
$$

Using a coordinate transformation to polar coordinates with

$$
x=r \cos \varphi \quad y=r \sin \varphi \quad \bar{x}=\bar{r} \cos \bar{\varphi} \quad \bar{y}=\bar{r} \sin \bar{\varphi}
$$

leads to

$$
\begin{aligned}
& w_{\text {point }}(r, \varphi)=\frac{\mathrm{i} q_{0}}{8 \widetilde{D} \widetilde{k}_{b}^{2}}\left(H_{0}^{(1)}\left(-\sqrt{r^{2}+\bar{r}^{2}-2 r \bar{r} \cos (\varphi-\bar{\varphi})} \widetilde{k}_{b}\right)\right. \\
& \left.+H_{0}^{(2)}\left(-\mathrm{i} \sqrt{r^{2}+\bar{r}^{2}-2 r \bar{r} \cos (\varphi-\bar{\varphi})} \widetilde{k}_{b}\right)\right)
\end{aligned}
$$

\section{Other axisymmetric loadings}

In this Section, the solutions for a constant ring load and a constant circular load, shown in Figs. 1a and 1b, are derived. Two different solution techniques are shown. The first technique uses the integration of the point force response given in Eq. (2.26), which was also used in (Matrinček, 1994) for the dynamic response of pavement structures. The second approach uses the Hankel transform defined in Eqs. (2.5) to obtain the results.
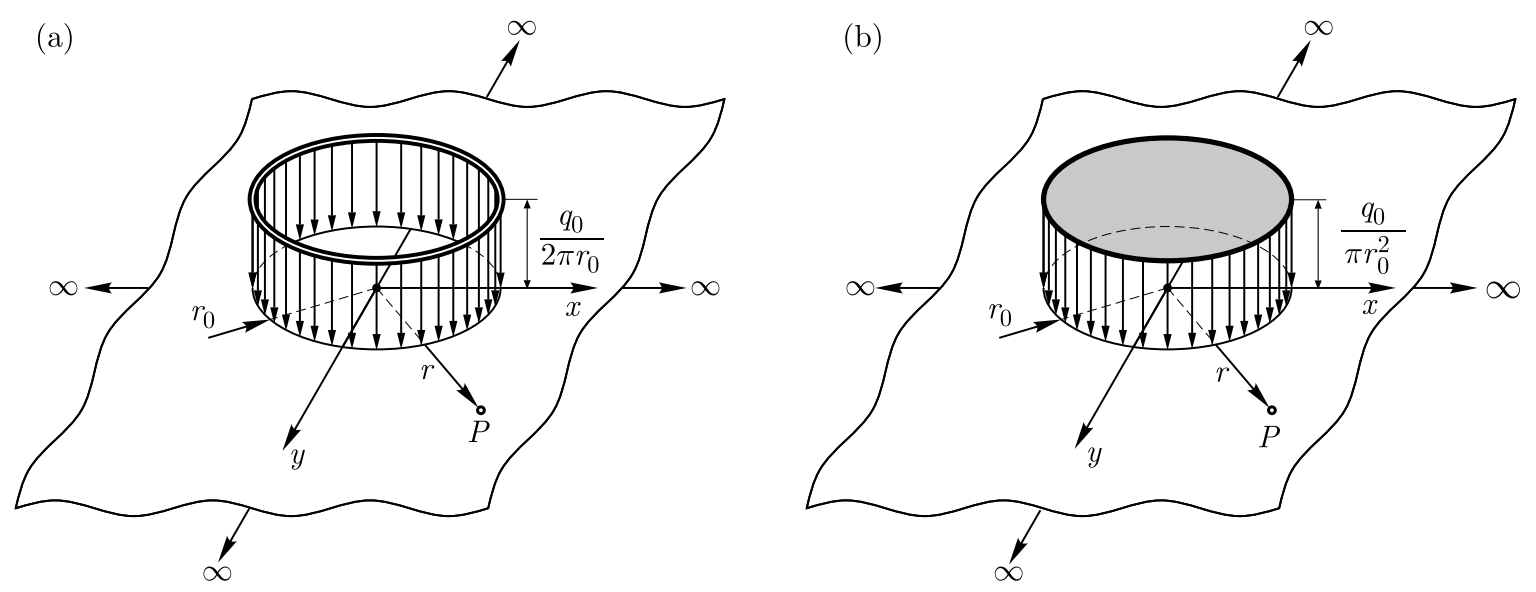

Fig. 1. Other axisymmetric load cases: (a) constant ring load, (b) constant circular load

\subsection{Constant ring load}

The constant ring load in Fig. 1a is given by

$$
q_{\text {ring }}(r)=\frac{q_{0}}{2 \pi r} \delta\left(r-r_{0}\right)
$$


with $\delta(\cdot)$ being the Dirac delta function, $r_{0}$ - radius of the ring load and $q_{0}$ - total external force. According to the theory of linear partial differential equations, the response to an arbitrary load can be found by integration of the point force solution (harmonic Green's function). This leads to

$$
\begin{aligned}
& w_{\text {ring }}(r, \varphi)=\int_{0}^{\infty}\left(\int_{0}^{2 \pi} q_{\text {ring }}(\bar{r}) w_{\text {point }}(r, \varphi, \bar{r}, \bar{\varphi}) \bar{r} d \bar{\varphi}\right) d \bar{r} \\
& =\frac{\mathrm{i} q_{0}}{16 \pi \widetilde{D}_{k_{b}^{2}}} \int_{0}^{\infty}\left(\int_{0}^{2 \pi} \frac{\delta\left(\bar{r}-r_{0}\right)}{\bar{r}}\left(H_{0}^{(1)}\left(-a \widetilde{k}_{b}\right)+H_{0}^{(2)}\left(-\mathrm{i} a \widetilde{k}_{b}\right)\right) \bar{r} d \bar{\varphi}\right) d \bar{r}
\end{aligned}
$$

with $a=\sqrt{r^{2}+\bar{r}^{2}-2 r \bar{r} \cos (\varphi-\bar{\varphi})}$. The integration with respect to $\bar{\varphi}$ can be carried out using the addition theorem for Bessel functions (Magnus et al., 1966)

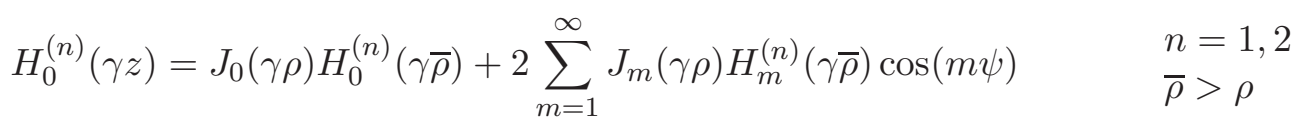

with $z=\sqrt{\rho^{2}+\bar{\rho}^{2}-2 \rho \bar{\rho} \cos \psi}$. Using that the integral

$$
\int_{0}^{2 \pi} \cos (m(\varphi-\bar{\varphi})) d \bar{\varphi}=0 \quad \text { for } \quad m=1,2, \ldots
$$

leads to

$$
w_{\text {ring }}(r)= \begin{cases}\frac{\mathrm{i} q_{0}}{8 \widetilde{D} \widetilde{k}_{b}^{2}} \int_{0}^{\infty} \delta\left(\bar{r}-r_{0}\right)\left(J_{0}\left(-\widetilde{k}_{b} \bar{r}\right) H_{0}^{(1)}\left(-\widetilde{k}_{b} r\right)+J_{0}\left(-\mathrm{i} \widetilde{k}_{b} \bar{r}\right) H_{0}^{(2)}\left(-\mathrm{i} \widetilde{k}_{b} r\right)\right) d \bar{r} & r>\bar{r} \\ \frac{\mathrm{i} q_{0}}{8 \widetilde{D} \widetilde{k}_{b}^{2}} \int_{0}^{\infty} \delta\left(\bar{r}-r_{0}\right)\left(J_{0}\left(-\widetilde{k}_{b} r\right) H_{0}^{(1)}\left(-\widetilde{k}_{b} \bar{r}\right)+J_{0}\left(-\mathrm{i} \widetilde{k}_{b} r\right) H_{0}^{(2)}\left(-\mathrm{i} \widetilde{k}_{b} \bar{r}\right)\right) d \bar{r} & r<\bar{r}\end{cases}
$$

Using the integral property of the dirac delta function gives the final result

$$
w_{\text {ring }}(r)= \begin{cases}\frac{\mathrm{i} q_{0}}{8 \widetilde{D} \widetilde{k}_{b}^{2}}\left(J_{0}\left(-\widetilde{k}_{b} r_{0}\right) H_{0}^{(1)}\left(-\widetilde{k}_{b} r\right)+J_{0}\left(-\mathrm{i} \widetilde{k}_{b} r_{0}\right) H_{0}^{(2)}\left(-\mathrm{i} \widetilde{k}_{b} r\right)\right) & r>r_{0} \\ \frac{\mathrm{i} q_{0}}{8 \widetilde{D} \widetilde{k}_{b}^{2}}\left(J_{0}\left(-\widetilde{k}_{b} r\right) H_{0}^{(1)}\left(-\widetilde{k}_{b} r_{0}\right)+J_{0}\left(-\mathrm{i} \widetilde{k}_{b} r\right) H_{0}^{(2)}\left(-\mathrm{i} \widetilde{k}_{b} r_{0}\right)\right) & r<r_{0}\end{cases}
$$

The function depends only on $r$, and it is obvious that the function is continuous at $r=r_{0}$.

Another possibility to get the result is the Hankel transform. The Hankel transform of the constant ring load is given by

$$
q_{\text {ring }}(r)=\frac{q_{0}}{2 \pi r} \delta\left(r-r_{0}\right) \mapsto \widetilde{q}_{\text {ring }}\left(k_{r}\right)=\frac{q_{0}}{2 \pi} J_{0}\left(k_{r} r_{0}\right)
$$

and combined with the transformed governing equation (Eq. (2.7)), the result in an integral form is given by

$$
w_{\text {ring }}(r)=\frac{q_{0}}{2 \pi \widetilde{D}} \int_{0}^{\infty} \frac{k_{r}}{k_{r}^{4}-\widetilde{k}_{b}^{4}} J_{0}\left(k_{r} r_{0}\right) J_{0}\left(k_{r} r\right) d k_{r}
$$


The result of this integral can be found by the general formula for integrals involving products of two Bessel functions (Lin, 2014)

$$
\int_{0}^{\infty} \frac{x^{\mu-\nu+2 \ell+1}}{x^{2}+a^{2}} J_{\mu}(p x) J_{\nu}(q x) d x=(-1)^{\ell} a^{\mu-\nu+2 \ell} K_{\mu}(p a) I_{\nu}(q a) \quad \ell \in \mathbb{N}_{0}
$$

with the restrictions

$$
-(\ell+1)<\operatorname{Re}(\mu)<\operatorname{Re}(\nu)-2 \ell+2 \text { and } p>q \text { and }-\frac{\pi}{2}<\arg (a)<\frac{\pi}{2}
$$

Choosing $\mu=0, \nu=0$ and $\ell=0$ and the partial fraction decomposition

$$
\frac{k_{r}}{k_{r}^{4}-\widetilde{k}_{b}^{4}}=\frac{1}{2 \widetilde{k}_{b}^{2}}\left(\frac{k_{r}}{k_{r}^{2}+\left(-\widetilde{k}_{b}^{2}\right)}-\frac{k_{r}}{k_{r}^{2}+\widetilde{k}_{b}^{2}}\right)
$$

leads to the final result

$$
w_{\text {ring }}(r)= \begin{cases}\frac{q_{0}}{4 \pi \widetilde{D} \widetilde{k}_{b}^{2}}\left(K_{0}\left(\widetilde{\mathrm{i}}_{b} r\right) I_{0}\left(\mathrm{i} \widetilde{k}_{b} r_{0}\right)-K_{0}\left(\widetilde{k}_{b} r\right) I_{0}\left(\widetilde{k}_{b} r_{0}\right)\right) & r>r_{0} \\ \frac{q_{0}}{4 \pi \widetilde{D} \widetilde{k}_{b}^{2}}\left(K_{0}\left(\widetilde{\mathrm{i}}_{b} r_{0}\right) I_{0}\left(\mathrm{i} \widetilde{k}_{b} r\right)-K_{0}\left(\widetilde{k}_{b} r_{0}\right) I_{0}\left(\widetilde{k}_{b} r\right)\right) & r<r_{0}\end{cases}
$$

Using the relations in Eqs. (2.21) and the relation (Abramowitz and Stegun, 1972)

$$
I_{0}(z)=J_{0}(\mathrm{i} z) \quad-\pi<\arg (z) \leqslant \frac{1}{2} \pi
$$

the results in Eqs. (3.6) become equivalent to Eqs. (3.12).

\subsection{Constant circular load}

The constant circular load in Fig. 1b is given by

$$
q_{\text {circ }}(r)=\frac{q_{0}}{r_{0}^{2} \pi} H\left(r_{0}-r\right)
$$

where $H(\cdot)$ is the Heaviside step function, $r_{0}$ - radius of the circular load and $q_{0}$ - total external force. The integration of the point load response leads to

$$
\begin{aligned}
& w_{\text {circ }}(r, \varphi)=\int_{0}^{\infty}\left(\int_{0}^{2 \pi} q_{\text {circ }}(\bar{r}) w_{\text {point }}(r, \varphi, \bar{r}, \bar{\varphi}) \bar{r} d \bar{\varphi}\right) d \bar{r} \\
& =\frac{\mathrm{i} q_{0}}{8 \pi r_{0}^{2} \widetilde{D}_{b}^{2}} \int_{0}^{\infty}\left(\int_{0}^{2 \pi} H\left(r_{0}-\bar{r}\right)\left(H_{0}^{(1)}\left(-a \widetilde{k}_{b}\right)+H_{0}^{(2)}\left(-\mathrm{i} a \widetilde{k}_{b}\right)\right) \bar{r} d \bar{\varphi}\right) d \bar{r}
\end{aligned}
$$

The integration with respect to $\bar{\varphi}$ is equivalent to integration from Eq. (3.2) to Eqs. (3.5). Using the property of the Heaviside step function and distinguishing between the case $r>r_{0}$ and $r<r_{0}$ leads to

$$
w_{\text {circ }}(r)= \begin{cases}\frac{\mathrm{i} q_{0}}{4 r_{0}^{2} \widetilde{D} \widetilde{k}_{b}^{2}} \int_{0}^{r_{0}}\left(J_{0}\left(-\widetilde{k}_{b} \bar{r}\right) H_{0}^{(1)}\left(-\widetilde{k}_{b} r\right)+J_{0}\left(-\mathrm{i} \widetilde{k}_{b} \bar{r}\right) H_{0}^{(2)}\left(-\mathrm{i} \widetilde{k}_{b} r\right)\right) \bar{r} d \bar{r} & r>r_{0} \\ \frac{\mathrm{i} q_{0}}{4 r_{0}^{2} \widetilde{D} \widetilde{k}_{b}^{2}}\left(\int_{0}^{r}\left(J_{0}\left(-\widetilde{k}_{b} \bar{r}\right) H_{0}^{(1)}\left(-\widetilde{k}_{b} r\right)+J_{0}\left(-\mathrm{i} \widetilde{k}_{b} \bar{r}\right) H_{0}^{(2)}\left(-\mathrm{i} \widetilde{k}_{b} r\right)\right) \bar{r} d \bar{r}\right. & \\ \left.\quad+\int_{r}^{r_{0}}\left(J_{0}\left(-\widetilde{k}_{b} r\right) H_{0}^{(1)}\left(-\widetilde{k}_{b} \bar{r}\right)+J_{0}\left(-\mathrm{i} \widetilde{k}_{b} r\right) H_{0}^{(2)}\left(-\mathrm{i} \widetilde{k}_{b} \bar{r}\right)\right) \bar{r} d \bar{r}\right) & r<r_{0}\end{cases}
$$


The recurrence formulas for Bessel and Hankel functions are given by (Watson, 1944)

$$
\begin{array}{ll}
\frac{d}{d z}\left(z J_{1}(z)\right)=z J_{0}(z) \quad \rightarrow \quad z J_{1}(z)=\int z J_{0}(z) d z \\
\frac{d}{d z}\left(z H_{1}^{(n)}(z)\right)=z H_{0}^{(n)}(z) \quad \rightarrow \quad z H_{1}^{(n)}(z)=\int z H_{0}^{(n)}(z) d z \quad n=1,2
\end{array}
$$

which allows the evaluation of the integrals in Eqs. (3.16). The final results are given by

$$
w_{\text {circ }}(r)= \begin{cases}\frac{-\mathrm{i} q_{0}}{4 r_{0} \widetilde{D} \widetilde{k}_{b}^{2}}\left(\frac{1}{\widetilde{k}_{b}} J_{1}\left(-\widetilde{k}_{b} r_{0}\right) H_{0}^{(1)}\left(-\widetilde{k}_{b} r\right)+\frac{1}{\mathrm{i} \widetilde{k}_{b}} J_{1}\left(-\mathrm{i} \widetilde{k}_{b} r_{0}\right) H_{0}^{(2)}\left(-\mathrm{i} \widetilde{k}_{b} r\right)\right) & r>r_{0} \\ \frac{-\mathrm{i} q_{0}}{4 r_{0}^{2} \widetilde{D} \widetilde{k}_{b}^{2}}\left(\frac{4}{\mathrm{i} \pi \widetilde{k}_{b}^{2}}+\frac{r_{0}}{\widetilde{k}_{b}} J_{0}\left(-\widetilde{k}_{b} r\right) H_{1}^{(1)}\left(-\widetilde{k}_{b} r_{0}\right)+\frac{r_{0}}{\mathrm{i} \widetilde{k}_{b}} J_{0}\left(-\mathrm{i} \widetilde{k}_{b} r\right) H_{1}^{(2)}\left(-\mathrm{i} \widetilde{k}_{b} r_{0}\right)\right) & r<r_{0}\end{cases}
$$

In the computation of Eq. (3.18) 2 , the formulas for Wronskian determinants of Bessel functions (Magnus et al., 1966)

$$
\begin{aligned}
& J_{1}(z) H_{0}^{(1)}(z)-J_{0}(z) H_{1}^{(1)}(z)=\frac{2}{\mathrm{i} \pi z} \\
& J_{1}(z) H_{0}^{(2)}(z)-J_{0}(z) H_{1}^{(2)}(z)=-\frac{2}{\mathrm{i} \pi z}
\end{aligned}
$$

are used.

The response of the plate to a constant circular load can also be derived using the Hankel transform. Inserting the Hankel transform of a constant circular load

$$
q_{\text {circ }}(r)=\frac{q_{0}}{r_{0}^{2} \pi} H\left(r_{0}-r\right) \mapsto \widetilde{q}_{c i r c}\left(k_{r}\right)=\frac{q_{0}}{r_{0} \pi k_{r}} J_{1}\left(k_{r} r_{0}\right)
$$

in transformed governing equation (Eq. (2.7)) leads to

$$
w_{\text {circ }}(r)=\frac{q_{0}}{r_{0} \pi \widetilde{D}} \int_{0}^{\infty} \frac{1}{k_{r}^{4}-\widetilde{k}_{b}^{4}} J_{1}\left(k_{r} r_{0}\right) J_{0}\left(k_{r} r\right) d k_{r}
$$

The integral in Eq. (3.21) can be evaluated using the general formula from Eq. (3.9). The partial fraction decomposition

$$
\frac{1}{k_{r}^{4}-\widetilde{k}_{b}^{4}}=\frac{1}{2 \widetilde{k}_{b}^{2}}\left(\frac{1}{k_{r}^{2}+\left(-\widetilde{k}_{b}^{2}\right)}-\frac{1}{k_{r}^{2}+\widetilde{k}_{b}^{2}}\right)
$$

and the parameters $\mu=0, \nu=1$ and $l=0$ lead to the result for $r>r_{0}$. The result for $r<r_{0}$ can be found by expanding the partial fraction decomposition in Eq. (3.22) to

$$
\frac{1}{2 \widetilde{k}_{b}^{2}}\left(\frac{1}{k_{r}^{2}+\left(-\widetilde{k}_{b}^{2}\right)}-\frac{1}{k_{r}^{2}+\widetilde{k}_{b}^{2}}\right)=\frac{1}{2 \widetilde{k}_{b}^{4}}\left(-2+\frac{k_{r}^{2}}{k_{r}^{2}+\left(-\widetilde{k}_{b}^{2}\right)}+\frac{k_{r}^{2}}{k_{r}^{2}+\widetilde{k}_{b}^{2}}\right)
$$

The integrals involving the rational functions can be evaluated using Eq. (3.9) with the parameters $\mu=1, \nu=0$ and $l=0$, while the integral with the constant factor is a discontinuous Weber-Schafheitlin integral (Watson, 1944)

$$
\int_{0}^{\infty} J_{0}(a t) J_{1}(b t) d t=\left\{\begin{array}{cc}
0 & b<a \\
\frac{1}{b} & b>a
\end{array}\right.
$$


The final results are

$$
\left.w_{\text {circ }}(r)\right)= \begin{cases}\frac{-q_{0}}{2 r_{0} \pi \widetilde{D} \widetilde{k}_{b}^{2}}\left(\frac{1}{\widetilde{k}_{b}} I_{1}\left(\widetilde{k}_{b} r_{0}\right) K_{0}\left(\widetilde{k}_{b} r\right)-\frac{1}{\mathrm{i} \widetilde{k}_{b}} I_{1}\left(\widetilde{\mathrm{i}}_{b} r_{0}\right) K_{0}\left(\mathrm{i} \widetilde{k}_{b} r\right)\right) & r>r_{0} \\ \frac{-q_{0}}{2 r_{0}^{2} \pi \widetilde{D} \widetilde{k}_{b}^{2}}\left(\frac{2}{\widetilde{k}_{b}^{2}}-\frac{r_{0}}{\widetilde{k}_{b}} I_{0}\left(\widetilde{k}_{b} r\right) K_{1}\left(\widetilde{k}_{b} r_{0}\right)+\frac{r_{0}}{\mathrm{i} \widetilde{k}_{b}} I_{0}\left(\widetilde{\mathrm{i}}_{b} r\right) K_{1}\left(\mathrm{i} \widetilde{k}_{b} r_{0}\right)\right) & r<r_{0}\end{cases}
$$

Using the relations between the Bessel functions, the results become equivalent to the results in Eqs. (3.18).

\section{Non-axisymmetric loading}

A closed-form solution for the out-of-plane displacement of a plate can also be calculated for certain types of non-axisymmetric loading by the integration of the point force response. Especially, the loading shown in Fig. 2 has a practical value, since it appears when a circular cylinder is mounted to a plate and loaded with a harmonic horizontal force (Korenev, 2002).

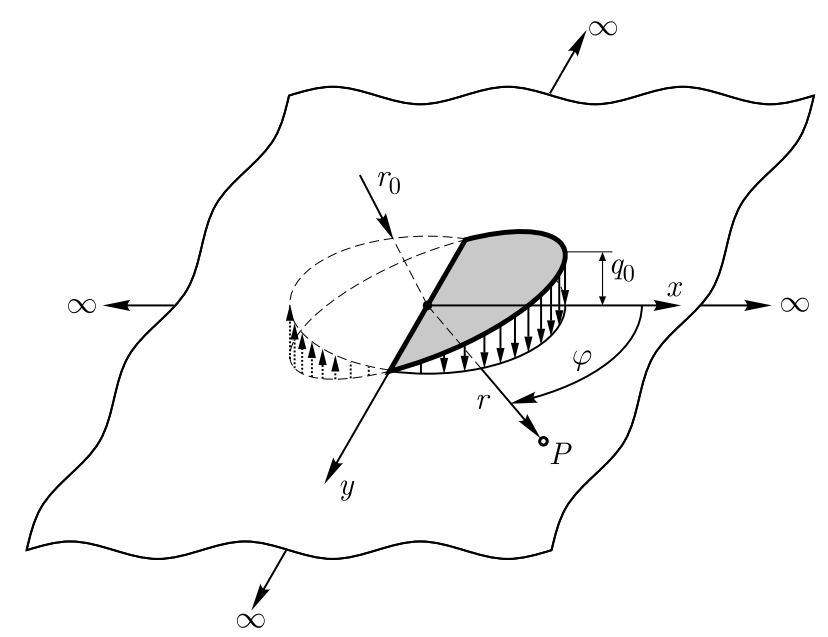

Fig. 2. Alternating circular load

The load shown in Fig. 2 is given in the polar coordinates by

$$
q_{\text {nonaxi }}(r, \varphi)=\frac{q_{0} r}{r_{0}} H\left(r_{0}-r\right) \cos \varphi .
$$

Integrating the point load response leads to

$$
\begin{aligned}
& w_{\text {nonaxi }}(r, \varphi)=\int_{0}^{\infty}\left(\int_{0}^{2 \pi} q_{\text {nonaxi }}(\bar{r}, \bar{\varphi}) w_{\text {point }}(r, \varphi, \bar{r}, \bar{\varphi}) \bar{r} d \bar{\varphi}\right) d \bar{r} \\
& =\frac{\mathrm{i} q_{0}}{8 r_{0} \widetilde{D} \widetilde{k}_{b}^{2}} \int_{0}^{\infty}\left(\int_{0}^{2 \pi} H\left(r_{0}-\bar{r}\right)\left(H_{0}^{(1)}\left(-a \widetilde{k}_{b}\right)+H_{0}^{(2)}\left(-\mathrm{i} a \widetilde{k}_{b}\right)\right) \bar{r}^{2} \cos \bar{\varphi} d \bar{\varphi}\right) d \bar{r}
\end{aligned}
$$

The integration with respect to $\bar{\varphi}$ can be carried out using the addition theorem for Bessel functions, given in Eq. (3.3) and the integral

$$
\int_{0}^{2 \pi} \cos \bar{\varphi} \cos (n(\varphi-\bar{\varphi})) d \bar{\varphi}= \begin{cases}\pi \cos \varphi & n=1 \\ 0 & n \in \mathbb{N}_{0} \mid n \neq 1\end{cases}
$$


Using the property of the Heaviside step function and distinguishing between the case $r>r_{0}$ and $r<r_{0}$, the integrals with respect to $\bar{r}$ are given by

$$
w_{\text {nonaxi }}(r, \varphi)=\left\{\begin{array}{cc}
\frac{\mathrm{i} q_{0} \pi \cos \varphi}{4 r_{0} \widetilde{D}_{k_{b}^{2}}^{2}} \int_{0}^{r_{0}}\left(J_{1}\left(-\widetilde{k}_{b} \bar{r}\right) H_{1}^{(1)}\left(-\widetilde{k}_{b} r\right)+J_{1}\left(-\mathrm{i} \widetilde{k}_{b} \bar{r}\right) H_{1}^{(2)}\left(-\mathrm{i} \widetilde{k}_{b} r\right)\right) \bar{r}^{2} d \bar{r} & r>r_{0} \\
\frac{\mathrm{i} q_{0} \pi \cos \varphi}{4 r_{0} \widetilde{D} \widetilde{k}_{b}^{2}}\left(\int_{0}^{r}\left(J_{1}\left(-\widetilde{k}_{b} \bar{r}\right) H_{1}^{(1)}\left(-\widetilde{k}_{b} r\right)+J_{1}\left(-\mathrm{i} \widetilde{k}_{b} \bar{r}\right) H_{1}^{(2)}\left(-\mathrm{i} \widetilde{k}_{b} r\right)\right) \bar{r}^{2} d \bar{r}\right. & (4.4) \\
\left.\quad+\int_{r}^{r_{0}}\left(J_{1}\left(-\widetilde{k}_{b} r\right) H_{1}^{(1)}\left(-\widetilde{k}_{b} \bar{r}\right)+J_{1}\left(-\mathrm{i} \widetilde{k}_{b} r\right) H_{1}^{(2)}\left(-\mathrm{i} \widetilde{k}_{b} \bar{r}\right)\right) \bar{r}^{2} d \bar{r}\right) & r<r_{0}
\end{array}\right.
$$

Using the recurrence formulas for Bessel and Hankel functions (Watson, 1944)

$$
\begin{aligned}
& \frac{d}{d z}\left(z^{2} J_{2}(z)\right)=z^{2} J_{1}(z) \quad \rightarrow \quad z^{2} J_{2}(z)=\int z^{2} J_{1}(z) d z \\
& \frac{d}{d z}\left(z^{2} H_{2}^{(n)}(z)\right)=z^{2} H_{1}^{(n)}(z) \quad \rightarrow \quad z^{2} H_{2}^{(n)}(z)=\int z^{2} H_{1}^{(n)}(z) d z \quad n=1,2
\end{aligned}
$$

and the formulas for Wronskian determinants of the Bessel functions given in Eqs. (3.19), the integration with respect to $\bar{r}$ leads to the final result

$$
w_{\text {nonaxi }}(r)=\left\{\begin{array}{cc}
\frac{-\mathrm{i} q_{0} \pi r_{0} \cos \varphi}{4 \widetilde{D}_{b}^{2}}\left(\frac{1}{\widetilde{k}_{b}} J_{2}\left(-\widetilde{k}_{b} r_{0}\right) H_{1}^{(1)}\left(-\widetilde{k}_{b} r\right)\right. & r>r_{0} \\
\left.+\frac{1}{\mathrm{i} \widetilde{k}_{b}} J_{2}\left(-\mathrm{i} \widetilde{k}_{b} r_{0}\right) H_{1}^{(2)}\left(-\mathrm{i} \widetilde{k}_{b} r\right)\right) & \\
\frac{-\mathrm{i} q_{0} \pi \cos \varphi}{4 \widetilde{D}_{k_{b}}^{2}}\left(\frac{4 r}{\mathrm{i} r_{0} \widetilde{k}_{b}^{2} \pi}+\frac{r_{0}}{\widetilde{k}_{b}} J_{1}\left(-\widetilde{k}_{b} r\right) H_{2}^{(1)}\left(-\widetilde{k}_{b} r_{0}\right)\right. & r<r_{0} \\
\left.+\frac{r_{0}}{\mathrm{i} \widetilde{k}_{b}} J_{1}\left(-\mathrm{i} \widetilde{k}_{b} r\right) H_{2}^{(2)}\left(-\mathrm{i} \widetilde{k}_{b} r_{0}\right)\right) &
\end{array}\right.
$$

\section{Limit absorption principle - the undamped plate}

The limit absorption principle states that a purely elastic solid is an idealization of a weakly absorbing material and, therefore, the solution of the undamped plate can be found by the limiting case $\eta \rightarrow 0$ and $\Phi \rightarrow 0$ (Filippi, 2010). Replacing $\widetilde{D} \rightarrow D$ and $\widetilde{k}_{b} \rightarrow k_{b}$ in Eqs. (2.20), (3.6), (3.18) and (4.6) gives the resulting displacement of the undamped plate. The solution for the point force excitation in Eq. (2.20) becomes identical to the solutions, which can be found in the literature, e.g. (Vanmaele et al., 2007) for the undamped plate.

\section{Numerical example}

In this Section, the harmonic response of an infinite aluminium plate with a Young's modulus $E=7 \cdot 10^{10} \mathrm{~N} / \mathrm{m}^{2}$, Poisson's ratio $\nu=0.3$, density $\rho=2790 \mathrm{~kg} / \mathrm{m}^{3}$, material loss factor $\eta=0.001$ and thickness $h=0.002 \mathrm{~m}$ is shown. For all load cases, the excitation frequency is $f=50 \mathrm{~Hz}$.

The real and imaginary part of the out-of-plane displacement $w$ of the plate excited by a harmonic point force $\left(q_{0}=1 \mathrm{~N}\right)$, harmonic constant ring load $\left(q_{0}=1 \mathrm{~N}, r_{0}=2.5 \mathrm{~m}\right)$, harmonic constant circular load $\left(q_{0}=1 \mathrm{~N}, r_{0}=2.5 \mathrm{~m}\right)$ and a harmonic alternating circular load $\left(q_{0}=1 \mathrm{~N}\right.$, $r_{0}=2.5 \mathrm{~m}$ ) is shown in Figs. 3-6. The point load solution shows the highest displacement amplitude at the center of the excitation $(r=0)$. 
(a)

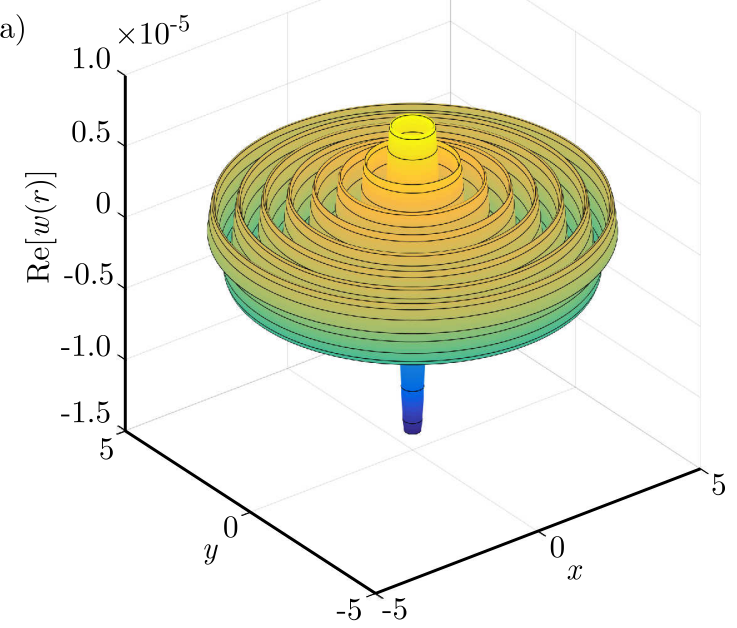

(b)

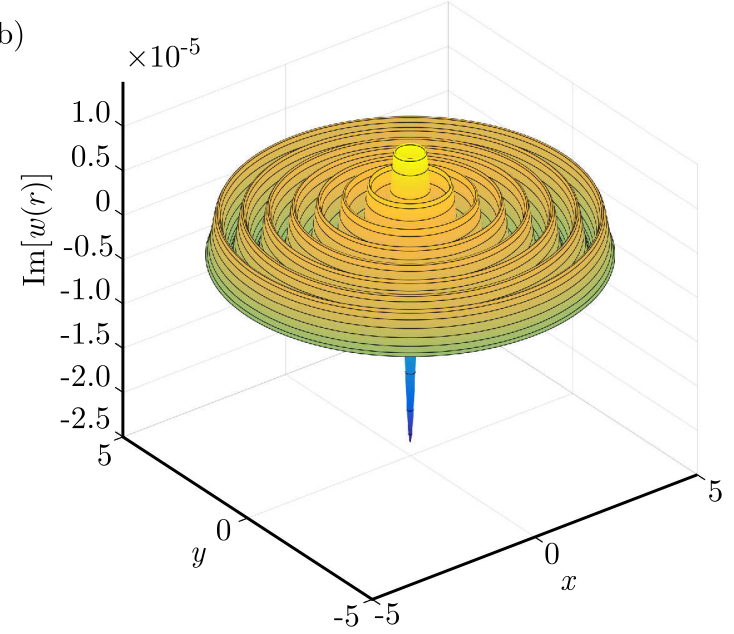

Fig. 3. Plate response to a harmonic point load: (a) real part of $w(r)$, (b) imaginary part of $w(r)$

(a)

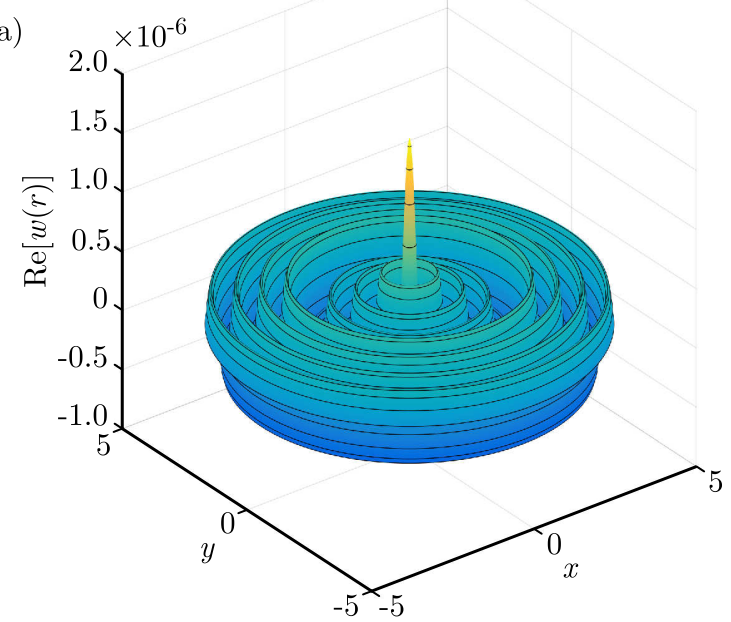

(b)

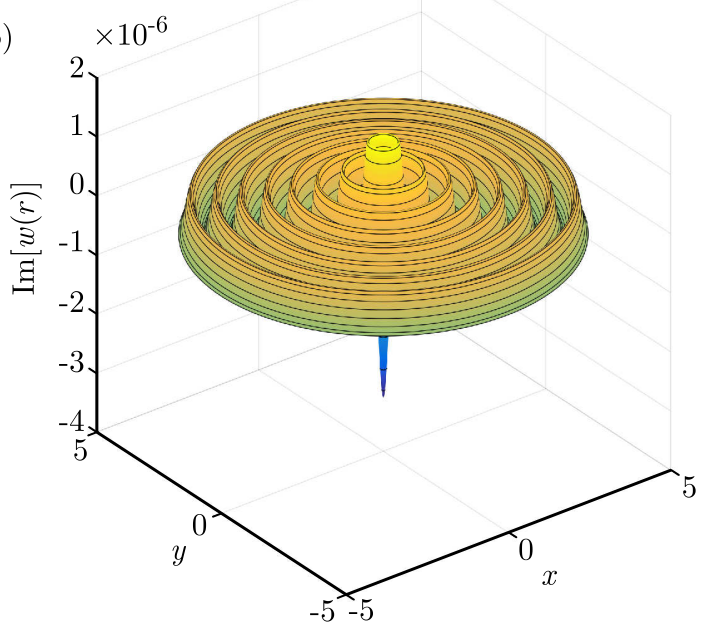

Fig. 4. Plate response to a harmonic ring load: (a) real part of $w(r)$, (b) imaginary part of $w(r)$

(a)

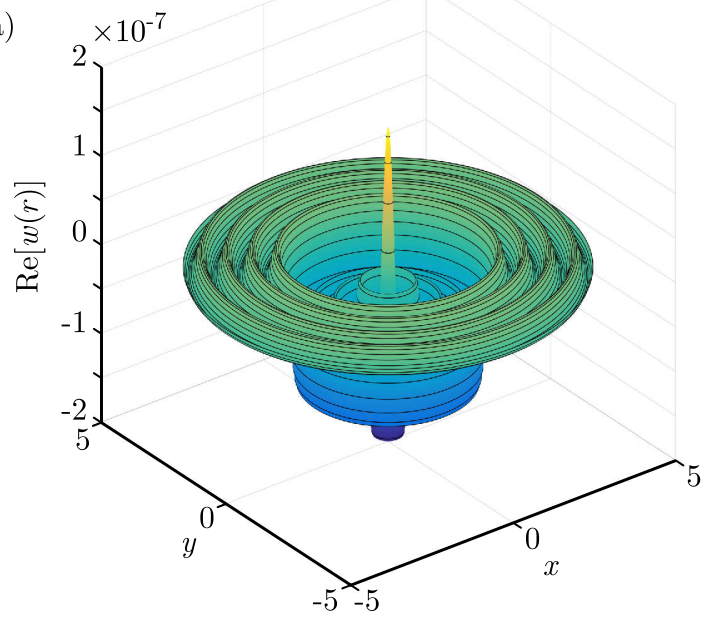

(b) $\times 10^{-7}$

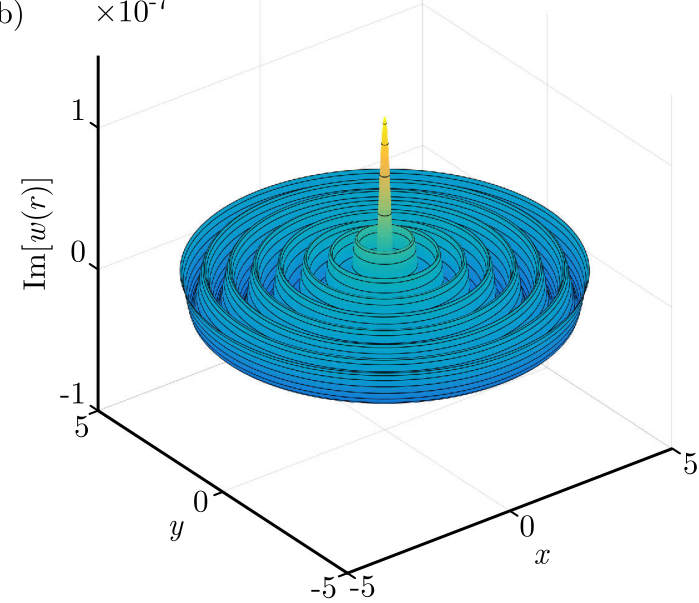

Fig. 5. Plate response to a harmonic circular load: (a) real part of $w(r)$, (b) imaginary part of $w(r)$ 

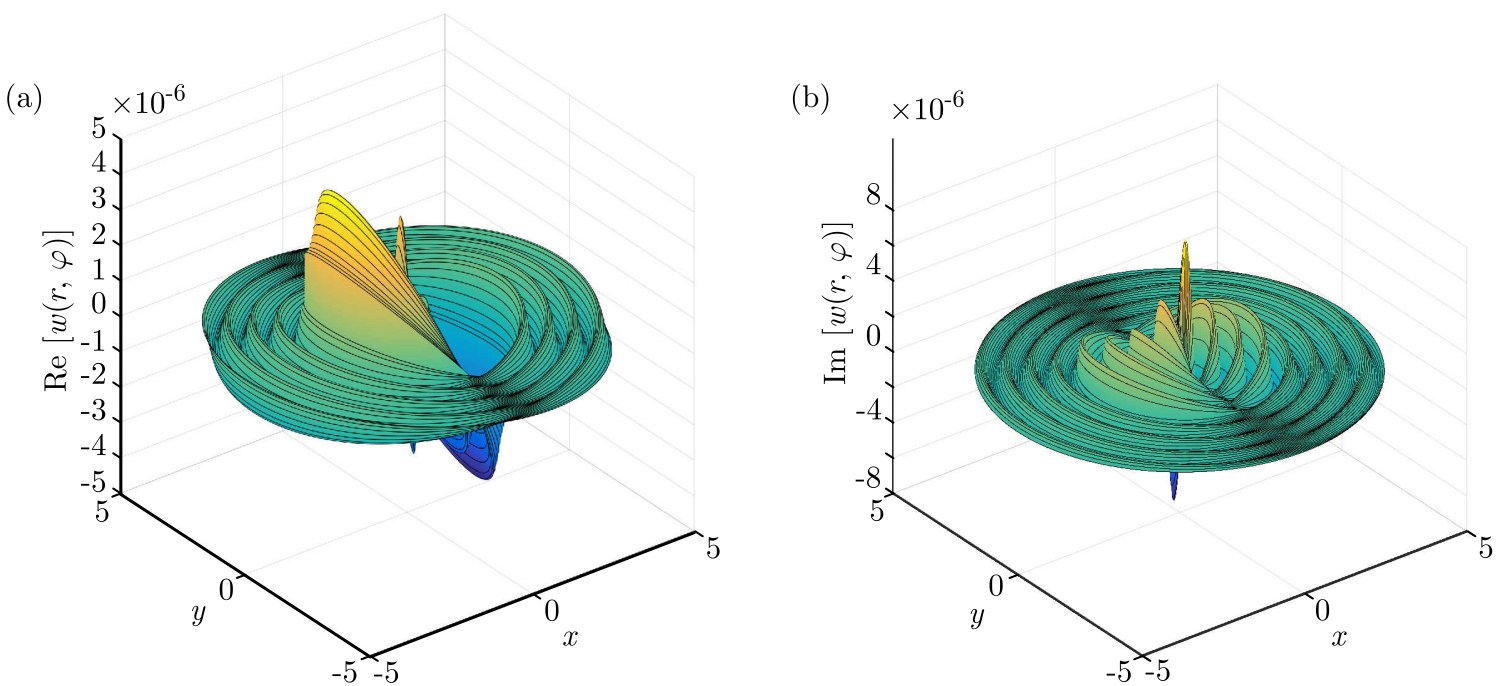

Fig. 6. Plate response to a harmonic alternating circular load: (a) real part of $w(r, \varphi)$,

(b) imaginary part of $w(r, \varphi)$

\section{Conclusion and future research topics}

New closed-form solutions for the harmonic vibrations of infinite Kirchhoff plates have been developed for different load cases. The response to a harmonic point load has been reviewed and two different techniques, the Hankel transform and the integration of the point load solution, have been used to calculate the response to a harmonic ring load, harmonic circular load and a harmonic alternating circular load. The new particular solutions can be used to extend the applicability of indirect Trefftz methods for the analysis of forced Kirchhoff plate vibrations.

\section{References}

1. Abramowitz M., Stegun I., 1972, Handbook of Mathematical Functions: with Formulas, Graphs, and Mathematical Tables, New York, Dover Publications

2. Bathe K., 2006, Finite Element Procedures, New Jersey, Prentice Hall

3. Chandrashekhara K., 2001, Theory of Plates, Hyderabad, Universities Press

4. Cremer L., Heckl M., Petersson B., 2005, Structure-Born Sound, Berlin, Springer Verlag

5. Debnath L., Bhatta D., 2014, Integral Transforms and Their Applications, Boca Raton, CRC Press

6. Filippi P.J.T., 2010, Vibrations and Acoustic Radiation of Thin Structures: Physical Basis, Theoretical Analysis and Numerical Methods, Hoboken, Wiley

7. Junger M.C., FeIt D., 1986, Sound, Structures, and their Interaction, Massachusetts, MIT Press

8. Kirchhoff B., 1850, Über das Gleichgewicht und die Bewegung einer elastischen Scheibe, Journal für die reine und angewandte Mathematik, 40, 51-88

9. Klanner M., Ellermann K., 2015, Wave Based Method for the steady-state vibrations of thick plates, Journal of Sound and Vibration, 345, 146-161

10. Korenev B., 2002, Bessel Functions and their Applications, London, Taylor \& Francis

11. LIN Q.-G., 2014, Infinite integrals involving Bessel functions by an improved approach of contour integration and the residue theorem, The Ramanujan Journal, 35, 3, 443-466 
12. Magnus W., Oberhettinger F., Soni R., 1966, Formulas and Theorems for the Special Functions of Mathematical Physics, Berlin, Springer Verlag

13. MartinčEK G., 1994, Dynamics of Pavement Structures, London, Taylor \& Francis

14. Mitrinović D., KeČKić J., 1984, The Cauchy Method of Residues, Dordrecht, D. Reidel Publishing Company

15. RAO S. 2007, Vibration of Continuous Systems, New Jersey, Wiley

16. Riou H., Ladevèze P., Kovalevsky L., 2013, The variational theory of complex rays: An answer to the resolution of mid-frequency 3D engineering problems, Journal of Sound and Vibration, 332, $1947-1960$

17. Rouch P., Ladevèze P., 2003, The variational theory of complex rays: A predictive tool for medium-frequency vibrations, Computer Methods in Applied Mechanics and Engineering, 192, 28/30, 3301-3315

18. Trefftz E., 1926, Ein Gegenstück zum Ritzschen Verfahren, Proceedings of the 2nd International Congress of Applied Mechanics, Zurich, Orell Fussli Verlag, 131-137

19. Vanmaele C., Vandepitte D., Desmet W., 2007, An efficient wave based prediction technique for plate bending vibrations, Computer Methods in Applied Mechanics and Engineering, 196, 31783189

20. Watson G., 1944, A Treatise on the Theory of Bessel Functions, London, Cambridge University Press

Manuscript received November 1, 2016; accepted for print April 5, 2017 